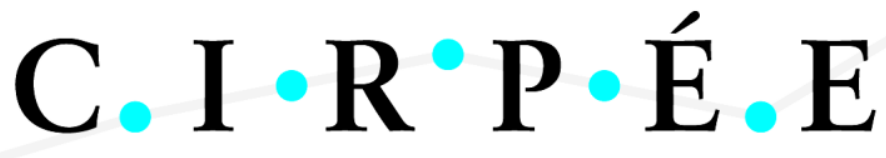

Centre Interuniversitaire sur le Risque, les Politiques Économiques et l'Emploi

Cahier de recherche/Working Paper 12-39

\section{Price Discrimination of Congestible Network Goods}

\author{
Maxime Agbo \\ Marc Santugini \\ Jonathan W. Williams
}

Octobre/October 2012

Agbo: Département d'économie, Agrocampus Ouest maxime.agbo@agrocampus-ouest.fr

Santugini: Institute of Applied Economics and CIRPÉE, HEC Montréal

marc.santugini@hec.ca

Williams: Department of Economics, University of Georgia

jonwms@uga.edu

We thank some North American Service Providers that participated in this project for sharing both data and industry expertise. 


\begin{abstract}
:
We study second-degree price discrimination for a congestible network good. We show that the seller does not always provide distinct contracts (i.e., it is not always optimal to price discriminate) and that it is more likely for the low-valuation buyer to be excluded. Because of the network externality through congestion, no buyer receives an efficient allocation. In particular, the high-valuation buyer might be offered a higher or a lower quality (relative to the first-degree price discrimination offer). Moreover, with congestion and for values of the parameters for which all types are serviced, consumer surplus under second-degree price discrimination may be greater than consumer surplus under no price discrimination.
\end{abstract}

Keywords: Congestion, Network, Price Discrimination

JEL Classification: D40, D62, D86, L14 


\section{Introduction}

This paper investigates the effect of monopolistic second-degree price discrimination of congestible network goods on behavior and payoffs, focusing on the market for residential broadband service (RBB). In broadband networks, congestion arises when the network is over-utilized, resulting in a degraded experience for consumers. The resulting interdependence of individual demands, via the existence of congestion externalities, limits the applicability of standard results from the literature on price discrimination to this market (Robinson, 1933; Schmalensee, 1981; Varian, 1985; Holmes, 1989).

The market for RBB is characterized by a unique set of features. Often in any local area, a single provider of high-speed broadband service practices second-degree price discrimination by offering consumers products differentiated along at least one vertical dimension (i.e., tiers). ${ }^{1}$ These vertical dimensions often include, but are not limited to, a provisioned speed (i.e. bandwidth in megabits per second, $\mathrm{Mb} / \mathrm{s}$ ) and possibly a usage allowance (i.e. permitted to consume a maximum number of gigabytes, GB, per month). Our model allows for a single vertical dimension and assumes that consumers' utility and usage is increasing in this vertical dimension, ultimately resulting in greater congestion. ${ }^{2}$

The prevalence of congestion in broadband networks depends on a number of factors besides this vertical dimension. ${ }^{3}$ In broadband networks, a small number of users (i.e., a neighborhood) share a fixed amount of available bandwidth at any time. If the network is inadequately provisioned for content requests, congestion can arise and lead to saturation of critical links in the

\footnotetext{
${ }^{1}$ Detailed information on the number of broadband providers is available from the FCC at http://transition.fcc.gov/wcb/iatd/comp.html/. Very few areas are serviced by more than one provider offering downstream connections exceeding $3 \mathrm{Mb} / \mathrm{s}$.

${ }^{2}$ Greater speed decreases the time required to access online content and allows the user to access bandwidth-intensive applications, while a greater usage allowance reduces the shadow price of consuming content throughout a billing cycle. Both aspects lead to increased usage of RBB (Varian, 2001).

${ }^{3}$ See Clarke (2009) for a more detailed discussion of the structure of broadband networks and precisely how congestion arises.
} 
network. ${ }^{4}$ This saturation can lead to delays in the transmission of data packets, or even dropping of data packets. A user's experience can deteriorate rapidly with the proportion of packets dropped (or delayed), with over $2 \%$ corresponding to a very poor experience. While the solution to congestion seems to have an obvious answer, "build a bigger pipe", it is not as simple as it seems. There are a number of difficulties in properly provisioning a broadband network. Over the past year alone, March 2011 to March 2012, average usage grew by over 50\%. Accurately forecasting this growth is a difficult problem, as the development of a single application (e.g. NetFlix) can dramatically alter growth in usage. The size and discrete nature of the investment required to split a network node makes responding quickly to such innovations difficult.

The small number and high geographical concentration of users along with the timing of usage in broadband networks also presents difficulties for planning network investment. For any given number of users on a node, typically between 300 and 500 in cable networks, it is difficult to predict total usage due to the large variance in usage across subscribers. The median user consumes about $30 \mathrm{~GB}$ of data per month, while users in the top $0.1 \%$ consume over 40 times (1.3 terabytes) as much. Figure 1 plots the average monthly usage (GB), by quantile, for over 1 million broadband subscribers, across all tiers and participating broadband providers. With such heterogeneity in usage, even a "last-mile" network infrastructure that is over-provisioned can quickly become congested with just a single heavy user moving into a neighborhood. The geographically localized nature of broadband also makes day-to-day usage very unpredictable due to local shocks that drive usage. Poor weather in a particular area, for example, may force individuals indoors and drive up usage significantly. Broadband networks must also accommodate the significant variation in usage throughout a day. Figure 2 shows that peak usage is between $7 \mathrm{pm}$ and $11 \mathrm{pm}$, when average usage is more than three times the average the rest of the day. Collectively, these difficulties in accurately provisioning a broadband network and the rapid growth in consumption, along

\footnotetext{
${ }^{4}$ Nodes that link users in a localized area to an interface, a link to the backbone of the internet, are the first place where congestion can arise.
} 
with pressures to minimize costs associated with infrastructure investment, suggest that congestion in these types of networks will remain into the foreseeable future.

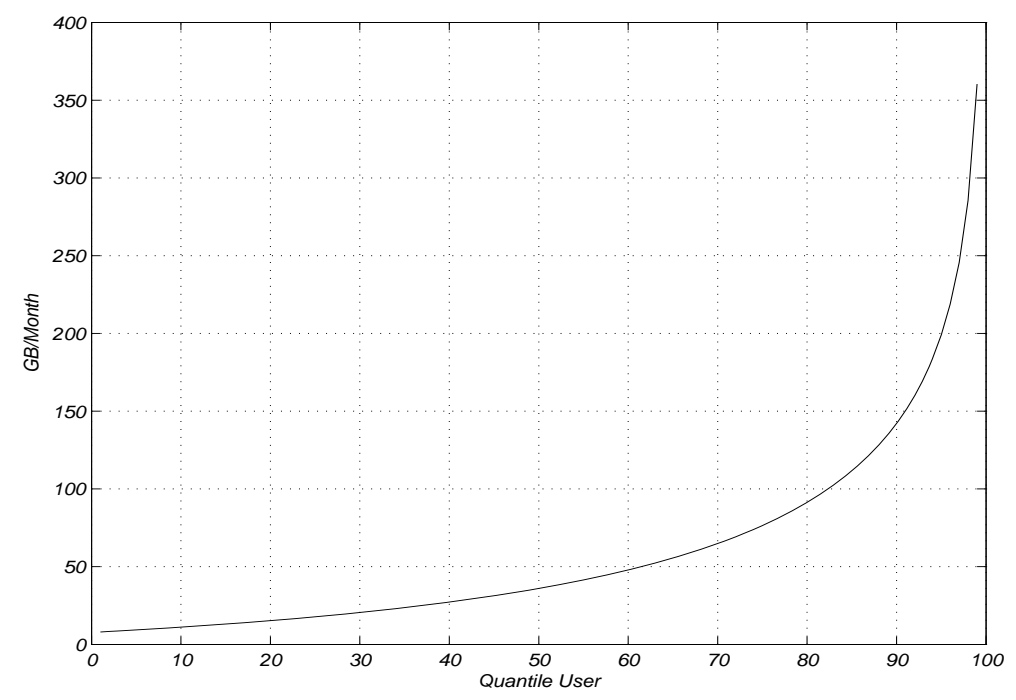

Figure 1: Average Usage by User Quantile

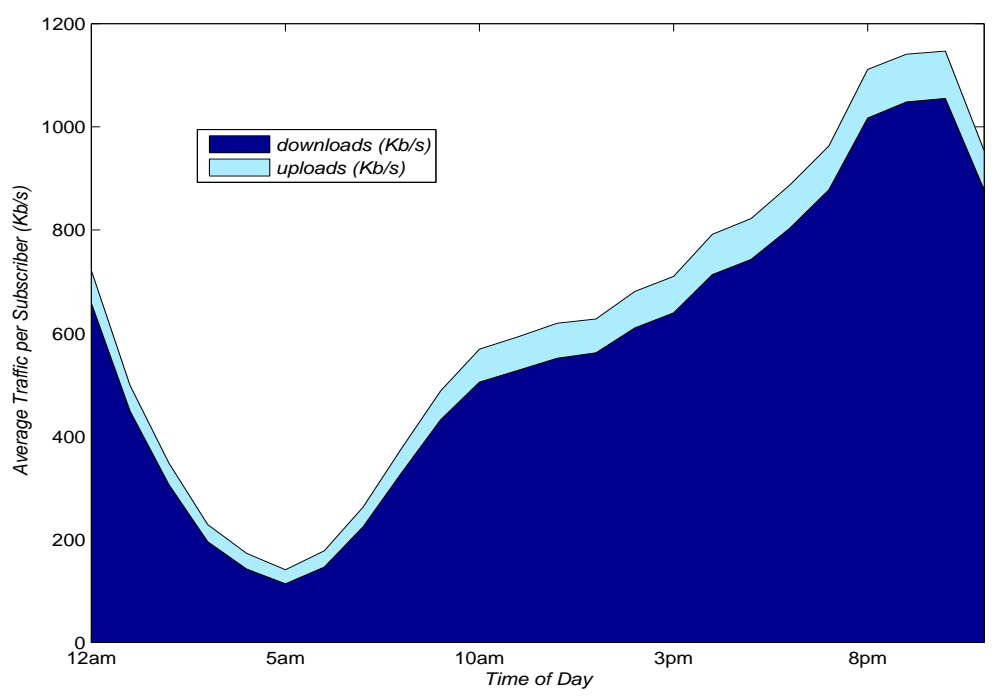

Figure 2: Traffic by Time of Day 
We build an analytically tractable model that captures the important features of the market for RBB: a small network of heterogeneous users facing congestion externalities, and a monopolistic provider that practices seconddegree price discrimination via packages that are vertically differentiated (e.g. usage allowance). After characterizing the unique optimal contract under second-degree price discrimination with congestion, we study the effect of second-degree price discrimination on behavior and welfare. ${ }^{5}$

We first show that congestion affects the seller's ability to offer distinct packages. If buyers are similar (but not identical) in their valuation of the good, the seller cannot discriminate and offers the same package to both buyers. We then show that congestion results in inefficient allocations for both buyers. In particular, the well-known result of "no distortion at the top" no longer holds once congestion externalities are introduced. The direction of the distortion depends on the values of the parameters. We then show that second-degree price discrimination may improve consumer surplus relative to a baseline of no price discrimination for certain parameter values. While this is true without congestion in situations in which the lower buyer is excluded under no price discrimination and is offered a package with price discrimination, we show that this is true even when both buyers are serviced. This runs counter to most arguments against price discrimination that focus on the redistribution of consumer surplus to the firm. This result arises due to the monopolist's willingness to degrade quality for users generating lowvalue traffic to limit the effects of congestion experienced by users generating high-value traffic.

The paper is organized as follows. Section 2 presents and discusses a model of second-degree price discrimination in which buyers face a congestion cost. Section 3 studies the effect of congestion on efficiency and consumer surplus. Section 4 concludes the paper.

\footnotetext{
${ }^{5}$ See Czerny and Zhang (2012) for a study on the impact of third-degree price discrimination by airlines on airports' welfare-optimal congestion charges.
} 


\section{Model and Optimal Behavior}

Consider a monopolistic provider of residential broadband (RBB). ${ }^{6}$ The good is assumed to be differentiated along a single vertical quality dimension. In the US, this single vertical dimension is often a usage allowance (e.g. allowance of 300GB per month) or provisioned speed (e.g. connection speed in $\mathrm{Mb} / \mathrm{s}$ ), which have been shown empirically to increase usage (Varian, 2001). ${ }^{7}$ Because the RBB framework is a relevant and important application, we now use the term usage allowance instead of quality for the remainder of the paper. Specifically, the seller offers a package with usage allowance $q \geq 0$ and charges a flat fee $t \geq 0$ for access to the network.

To account for the fact that buyers are part of small networks and have different valuations for internet services, we suppose that there are only two heterogeneous buyers. Buyer $L$ has a lower valuation for the internet service than buyer $H$. Each buyer derives utility from access to the network, but also receives disutility from the congestion in the network. If buyers $L$ and $H$ accept packages $\left\{q_{L}, t_{L}\right\}$ and $\left\{q_{H}, t_{H}\right\}$, respectively, net utility of buyer $L$ is

$$
u_{L}=\alpha q_{L}-q_{L}^{2}-c\left(q_{L}+q_{H}\right) q_{L}
$$

while net utility of buyer $H$ is

$$
u_{H}=q_{H}-q_{H}^{2}-c\left(q_{L}+q_{H}\right) q_{H},
$$

where the parameter $\alpha \in(0,1)$ reflects heterogeneity among buyers. The quadratic specification for utility is similar to that of Lambrecht et al. (2007). The average congestion cost $c\left(q_{L}+q_{H}\right)$ is increasing in total usage $q_{L}+q_{H}$, and the parameter $c \geq 0$ measures the degree of disutility due to congestion.

Because the seller is unable to perfectly price discriminate, we consider optimal behavior of the seller under second-degree price discrimination. The

\footnotetext{
${ }^{6}$ Markets are often local monopolies. See the FCC's National Broadband Plan at http://download.broadband.gov/plan/national-broadband-plan.pdf.

${ }^{7}$ Increased speed expands the set of applications and reduces the time required to access content, while a higher usage allowance relaxes the shadow price of consumption throughout a billing cycle.
} 
seller proposes two packages so that each type of buyer prefers the package intended for him. Definition 2.1 presents the seller's maximization problem under second-degree price discrimination. There are two sorts of constraints. The incentive compatibility constraints state that each buyer prefers the package designed for him. The individual rationality constraints ensure that each buyer accepts his designated package.

Definition 2.1. Under second-degree price discrimination, the seller solves the following program:

$$
\Pi^{* *}(\alpha, c)=\max _{q_{L}, q_{H}, t_{L}, t_{H} \geq 0} t_{L}+t_{H}
$$

subject to the incentive compatibility (IC) and individual rationality (IR) constraints, i.e.,

$$
\begin{aligned}
& (\underline{I C}): \alpha q_{L}-q_{L}^{2}-c\left(q_{L}+q_{H}\right) q_{L}-t_{L} \geq \alpha q_{H}-q_{H}^{2}-c\left(q_{H}+q_{H}\right) q_{H}-t_{H} \\
& (\overline{I C}): q_{H}-q_{H}^{2}-c\left(q_{L}+q_{H}\right) q_{H}-t_{H} \geq q_{L}-q_{L}^{2}-c\left(q_{L}+q_{L}\right) q_{L}-t_{L}
\end{aligned}
$$

and

$$
\begin{aligned}
& (\underline{I R}): \alpha q_{L}-q_{L}^{2}-c\left(q_{L}+q_{H}\right) q_{L} \geq t_{L}, \\
& (\overline{I R}): q_{H}-q_{H}^{2}-c\left(q_{L}+q_{H}\right) q_{H} \geq t_{H} .
\end{aligned}
$$

To ensure the existence of an interior solution, we assume that the congestion cost is not too high. ${ }^{8}$ Assumption 2.2 holds for the remainder of the paper.

Assumption 2.2. $c \in\left[0, \frac{2}{9}(1+\sqrt{10})\right]$, such that $4(1+c)-9 c^{2}>0$.

Proposition 2.3 provides optimal packages under second-degree price discrimination. The types of packages offered to the buyers depend on the relative valuation of buyer $L$ and the congestion cost parameter. The optimal packages stated in Proposition 2.3 embed the standard case with no congestion, i.e., $c=0$. Throughout the paper, we study the effect of congestion by comparing our results to the benchmark case $c=0$.

\footnotetext{
${ }^{8}$ See Footnote 19 in Appendix A.
} 
Proposition 2.3. Under second-degree price discrimination,

1. For $\alpha \in\left(0, \frac{2+5 c}{4(1+c)}\right]$, the seller offers $\left\{q_{L}^{* *}, t_{L}^{* *}\right\}=\{0,0\}$ and

$$
\left\{q_{H}^{* *}, t_{H}^{* *}\right\}=\left\{\frac{1}{2(1+c)}, \frac{1}{4(1+c)}\right\} .
$$

2. For $\alpha \in\left(\frac{2+5 c}{4(1+c)}, \frac{2(1+2 c)}{2+5 c}\right)$, the seller offers ${ }^{9}$

$$
q_{L}^{* *}=\frac{2(2 \alpha-1)-(5-4 \alpha) c}{4(1+c)-9 c^{2}},
$$

and

$$
q_{H}^{* *}=\frac{2-3(2 \alpha-1) c}{4(1+c)-9 c^{2}} .
$$

3. For $\alpha \in\left[\frac{2(1+2 c)}{2+5 c}, 1\right), c>0$ the seller offers the same package to both buyers,

$$
\left\{q^{* *}, t^{* *}\right\}=\left\{\frac{\alpha}{2(1+2 c)}, \frac{\alpha^{2}}{4(1+2 c)}\right\},
$$

Proof. See Appendix A.

To see more clearly how optimal usage allowances are influenced by the parameters of the model, we plot $q_{L}^{* *}$ and $q_{H}^{* *}$ as functions of $\alpha$ and $c$. Figure 3 provides both a contour plot and a three-dimensional view of $q_{L}^{* *}$ and $q_{H}^{* *}$. The three-dimensional view gives information about the magnitude of the effects of the parameters on optimal usage allowances. For any buyer, regardless of congestion (and as long as a buyer is offered a positive usage allowance), usage allowance is decreasing in the congestion cost. Moreover, an increase in buyer $L$ 's valuation increases his usage allowance.

\footnotetext{
${ }^{9}$ The expressions for $t_{L}^{* *}$ and $t_{H}^{* *}$ are cumbersome and omitted for this case. See Appendix A.
} 
Figure 3a: Contour of $q_{L}^{* *}$

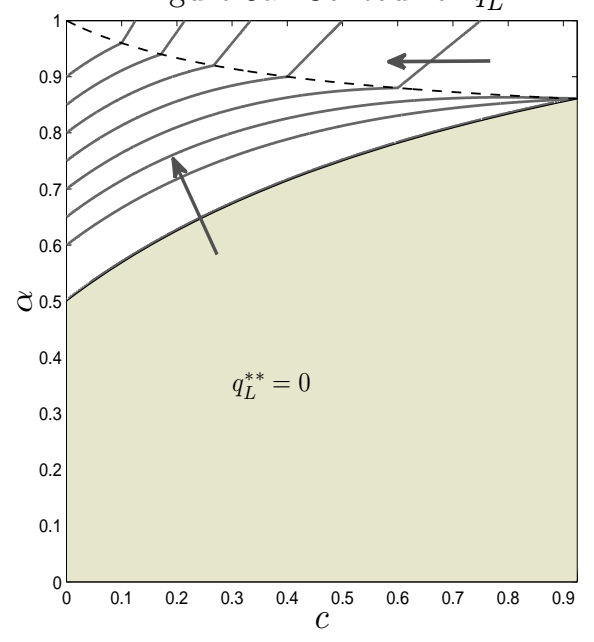

Figure 3c: $3 \mathrm{D}$ for $q_{L}^{* *}$

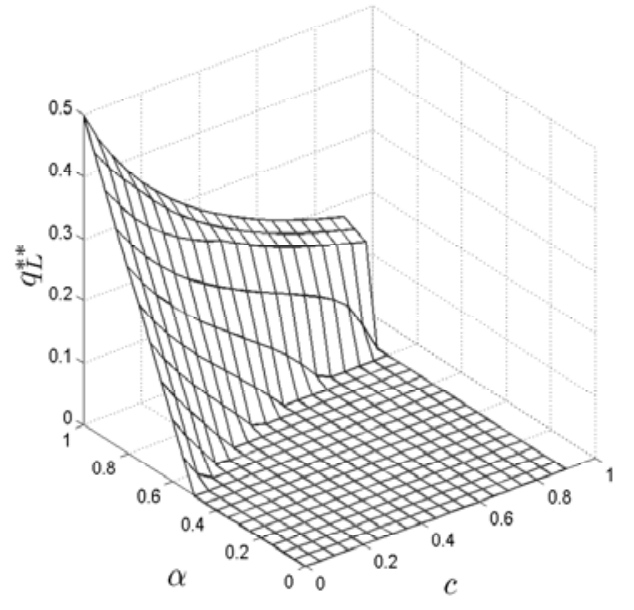

Figure 3b: Contour of $q_{H}^{* *}$

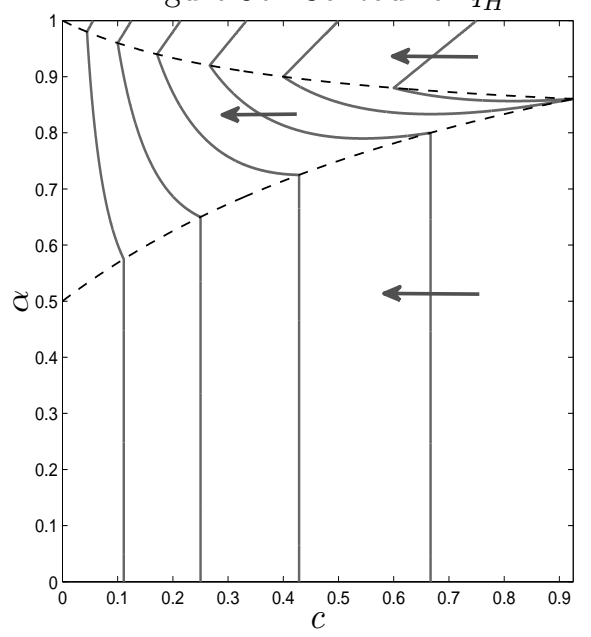

Figure 3d: 3D for $q_{H}^{* *}$

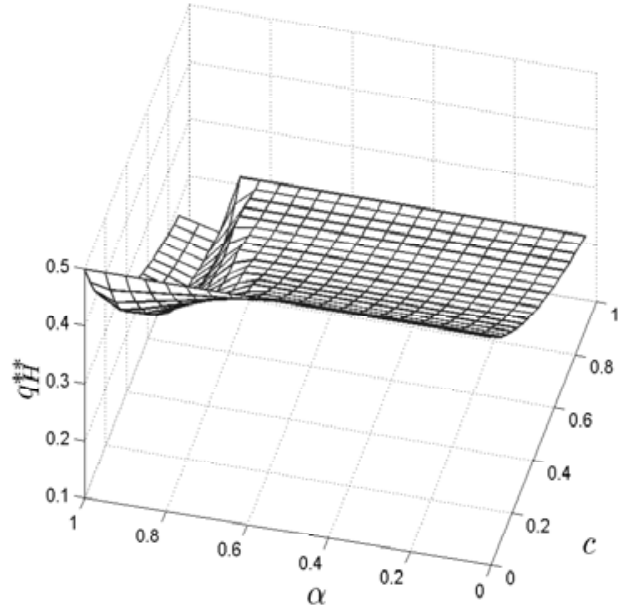

Figure 3: Optimal Usage Allowances. For Figures 3a and b, a contour plot reads similarly to an indifference curve in a utility graph. A curve on the graph regroups the set of pairs $\{\alpha, c\}$ that yields identical optimal allowances. The arrows indicates the direction for an increase in the value of usage allowances. Figures $3 \mathrm{c}$ and $\mathrm{d}$ complement Figures $3 \mathrm{a}$ and $\mathrm{b}$ by providing a three-dimensional view of usage allowances. 
Congestion adds a link between buyer L's valuation and the usage allowance received by buyer $H$. Indeed, when both buyers are offered positive usage allowances, then an increase in buyer $L$ 's valuation reduces the usage allowance offered to buyer $H$. Indeed, while an increase in $\alpha$ induces the seller to offer a higher usage allowance to buyer $L$, it also increases congestion cost for both buyers, which reduces the usage allowance offered to buyer $H$.

Remark 2.4. From Proposition 2.3, for $c>0$ and $\alpha \in\left(\frac{2+5 c}{4(1+c)}, \frac{2(1+2 c)}{2+5 c}\right)$, $\frac{\partial q_{H}^{* *}}{\partial \alpha}<0$

Having characterized analytically and graphically the optimal packages offered by the seller, we now discuss how congestion affects the seller's decision to effectively price discriminate (i.e., to offer distinct packages) and to service both buyers. Graphically, from Figure 4, the set of values for $\alpha$ for which the seller offers different and positive usage allowances is shrinking in $c$ and vanishes for large values of $c$.

First, Remark 2.5 states that the presence of congestion (i.e., $c>0$ ) affects the seller's ability to offer distinct packages. Although the seller never offers a greater usage allowance to the low-valuation buyer, it could be the same. ${ }^{10}$ Consistent with Proposition 2.3, under second-degree price discrimination with a congestion cost, the seller cannot offer two distinct qualities for higher values of $\alpha$, and hence $q_{L}^{* *}=q_{H}^{* *}$. If buyers are similar (but not identical) in their valuation of the good, the seller cannot discriminate and offers the same package to both buyers. For intermediate values of $\alpha$, the seller does offer two different packages while servicing both buyers, i.e., $q_{H}^{* *}>q_{L}^{* *}$. Finally, for low values of $\alpha$, the seller excludes the low-valuation buyer.

\footnotetext{
${ }^{10}$ As depicted in Figures 8 and 9 in Appendix D, an increase in the parameter for congestion cost or an increase in buyer $L$ 's valuation reduces the difference between usage allowances. While the seller never offers a better usage allowance to buyer $L$, the distance between the two usage allowances depends on buyer $L$ 's valuation and the congestion cost parameter.
} 


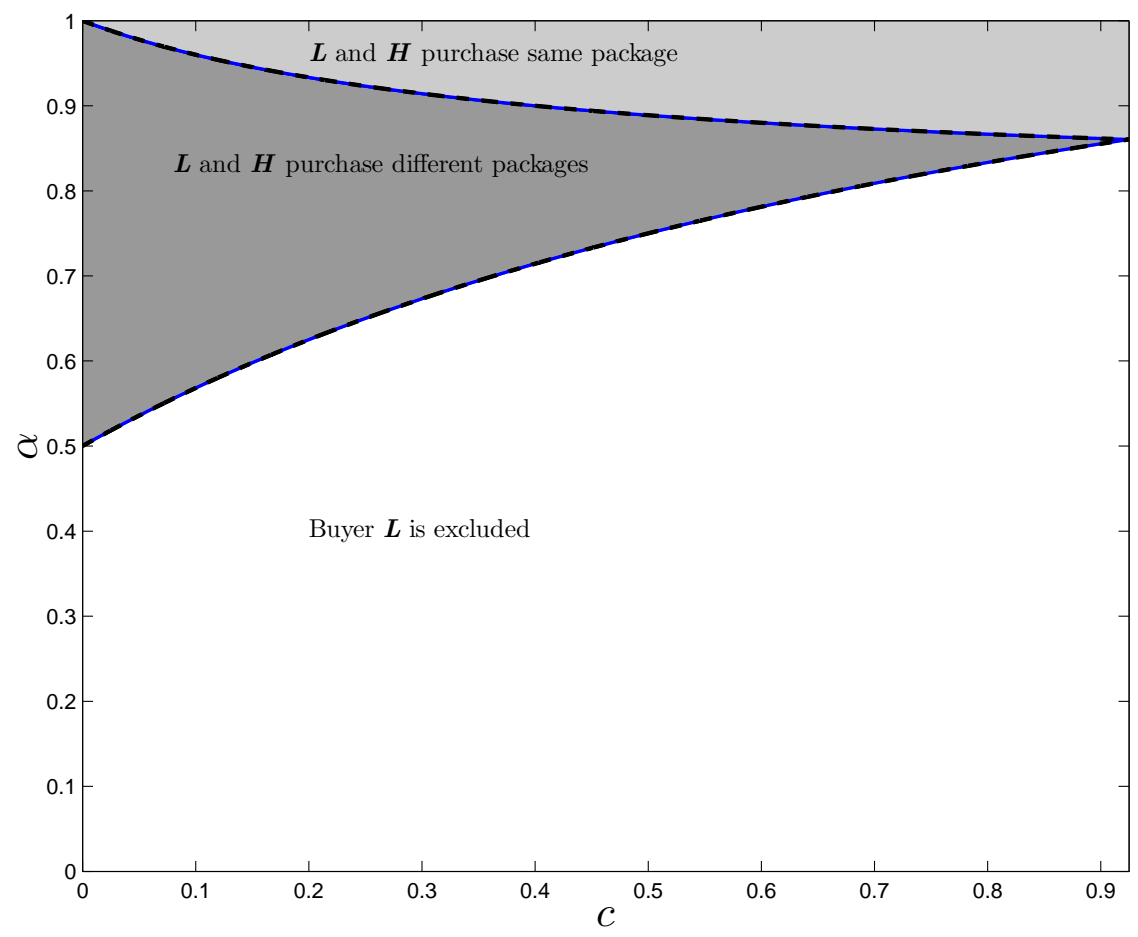

Figure 4: Types of Packages. The seller offers different types of packages depending on the values for the pair $\{\alpha, c\}$. The dotted lines separate the three cases. Consistent with Proposition 2.3, the increasing concave dotted line is $\alpha=\frac{2+5 c}{4(1+c)}$, while the decreasing convex dotted line is $\alpha=\frac{2(1+2 c)}{2+5 c}$. The area buyer $L$ is excluded regroups the set of pairs $\{\alpha, c\}$ for which $q_{H}^{* *}>q_{L}^{* *}=0$. The area $L$ and $H$ consume different packages regroups the set of pairs $\{\alpha, c\}$ for which $q_{H}^{* *}>q_{L}^{* *}>0$. The area $L$ and $H$ consume same package regroups the set of pairs $\{\alpha, c\}$ for which $q_{H}^{* *}=q_{L}^{* *}>0$. 
Remark 2.5. From Proposition 2.3, $0 \leq q_{L}^{* *} \leq q_{L}^{* *}<1 / 2$. Moreover, for $c>0$

1. for $\alpha \in\left(0, \frac{2(1+2 c)}{2+5 c}\right)$ two different packages are offered.

2. For $\alpha \in\left[\frac{2(1+2 c)}{2+5 c}, 1\right)$, only one package is offered to both buyers.

To explain the seller's decision to offer the same package with heterogeneous buyers, recall Remark 2.4. When there is congestion and both buyers are serviced, the usage allowance offered to buyer $H$ is decreasing in buyer $L$ 's valuation. For high enough values of $\alpha$, this negative externality on buyer $H$ implies that usage allowance are arbitrarily close to each other so that the seller offers only one type of package to both buyers. ${ }^{11}$ See also Figure 8 in Appendix D.

Remark 2.6 states that the seller's ability to serve both buyers depends on the congestion cost. An increase in the congestion cost $c$ increases the threshold for buyer $L$ to be excluded. See also Figure 9 in Appendix D.

Remark 2.6. From Proposition 2.3, an increase in the congestion cost makes it more likely for buyer $L$ to be excluded, i.e., it increases the range of values for $\alpha$ for which buyer $L$ is excluded.

\section{Effect of Congestion}

Having characterized and discussed how the seller's behavior changes with congestion, we now study the effect of congestion cost on efficiency and consumer surplus.

\subsection{Efficiency}

We show that a positive cost of congestion has a profound effect on efficiency, i.e., in general no buyer receives the efficient allocation. We begin by

\footnotetext{
${ }^{11}$ Offering more usage allowance to buyer $L$ than to buyer $H$ is not optimal for the seller. See Appendix A.
} 
providing the optimal usage allowances under first-degree (perfect) price discrimination in Proposition 3.1. We then compare perfect and second-degree price discrimination.

Proposition 3.1. Under perfect price discrimination, $q_{H}^{*}>q_{L}^{*} \geq 0$ such that

1. For $\alpha \in\left(0, \frac{c}{1+c}\right], q_{L}^{*}=0$ and

$$
q_{H}^{*}=\frac{1}{2(1+c)}
$$

2. For $\alpha \in\left(\frac{c}{1+c}, 1\right)$,

$$
\begin{aligned}
q_{L}^{*} & =\frac{\alpha-(1-\alpha) c}{2(1+2 c)}, \\
q_{H}^{*} & =\frac{1+(1-\alpha) c}{2(1+2 c)} .
\end{aligned}
$$

Proof. See Appendix B for a full-characterization and the proof for the case of perfect price discrimination.

Proposition 3.2 states that when the seller services both buyers under perfect and second-degree price discrimination, there is in general a distortion for buyer $H .{ }^{12}$ The inefficiency due to congestion can either increase of decrease usage allowance depending on the values of the parameters.

\footnotetext{
${ }^{12}$ When buyer $L$ is excluded under second-degree price discrimination, then $q_{H}^{* *}>q_{H}^{*}$ and $0=q_{L}^{* *} \leq q_{L}^{*}$ with equality in some cases.
} 
Proposition 3.2. Suppose that the seller services both buyer under perfect and second-degree price discrimination, i.e., $\alpha \in\left(\frac{2+5 c}{4(1+c)}, 1\right)$. Then, for $c>$ $0,{ }^{13}$

1. For $\alpha \in\left(\frac{2+5 c}{4(1+c)}, \frac{17 c+9 c^{2}+6}{20 c+9 c^{2}+8}\right), q_{H}^{* *}>q_{H}^{*}$.

2. For $\alpha=\frac{17 c+9 c^{2}+6}{20 c+9 c^{2}+8}, q_{H}^{* *}=q_{H}^{*}$.

3. For $\alpha \in\left(\frac{17 c+9 c^{2}+6}{20 c+9 c^{2}+8}, 1\right), q_{H}^{* *}<q_{H}^{*}$.

Proof. From Propositions 2.3 and 3.1, comparing (10) and (14) yields the conditions stated in Proposition 3.2.

Proposition 3.3 states the direction of the inefficiency for buyer $L$ 's usage allowance. When the seller offers the same package to both buyers under second-degree price discrimination, the distortion is downward (i.e., $q_{L}^{* *}<$ $\left.q_{L}^{*}\right)$. However, when the seller offers different packages under second-degree price discrimination, the distortion is upward (i.e., $q_{L}^{* *}>q_{L}^{*}$ ).

Proposition 3.3. Suppose that the seller services both buyers under perfect and second-degree price discrimination, i.e., $\alpha \in\left(\frac{2+5 c}{4(1+c)}, 1\right)$. Then, for $c>$ $0,{ }^{14}$

1. For $\alpha \in\left(\frac{2+5 c}{4(1+c)}, \frac{2 * 1+2 c)}{2+5 c}\right), q_{L}^{* *}<q_{L}^{*}$.

2. For $\alpha \in\left(\frac{2 * 1+2 c)}{2+5 c}, 1\right), q_{L}^{* *}>q_{L}^{*}$.

Proof. From Propositions 2.3 and 3.1, comparing (9) and (13) yields the conditions stated in Proposition 3.3.

The conditions stated in Propositions 3.2 and 3.3 are depicted in Figures 5 and 6. Specifically, Figures 5 and 6 orders the pair $\left\{q_{H}^{* *}, q_{H}^{*}\right\}$ and $\left\{q_{H}^{* *}, q_{H}^{*}\right\}$, respectively for different values of the pair $\{\alpha, c\} .{ }^{15}$ As in Figure 4, the dotted lines represent the boundaries for the different types of packages offered

\footnotetext{
${ }^{13}$ The conditions stated below are not valid when evaluated at $c=0$.

${ }^{14}$ The conditions stated below are not valid when evaluated at $c=0$.

${ }^{15}$ See Figures 10 and 11 in Appendix D for the size of these differences for buyer $H$ and buyer $L$, respectively.
} 


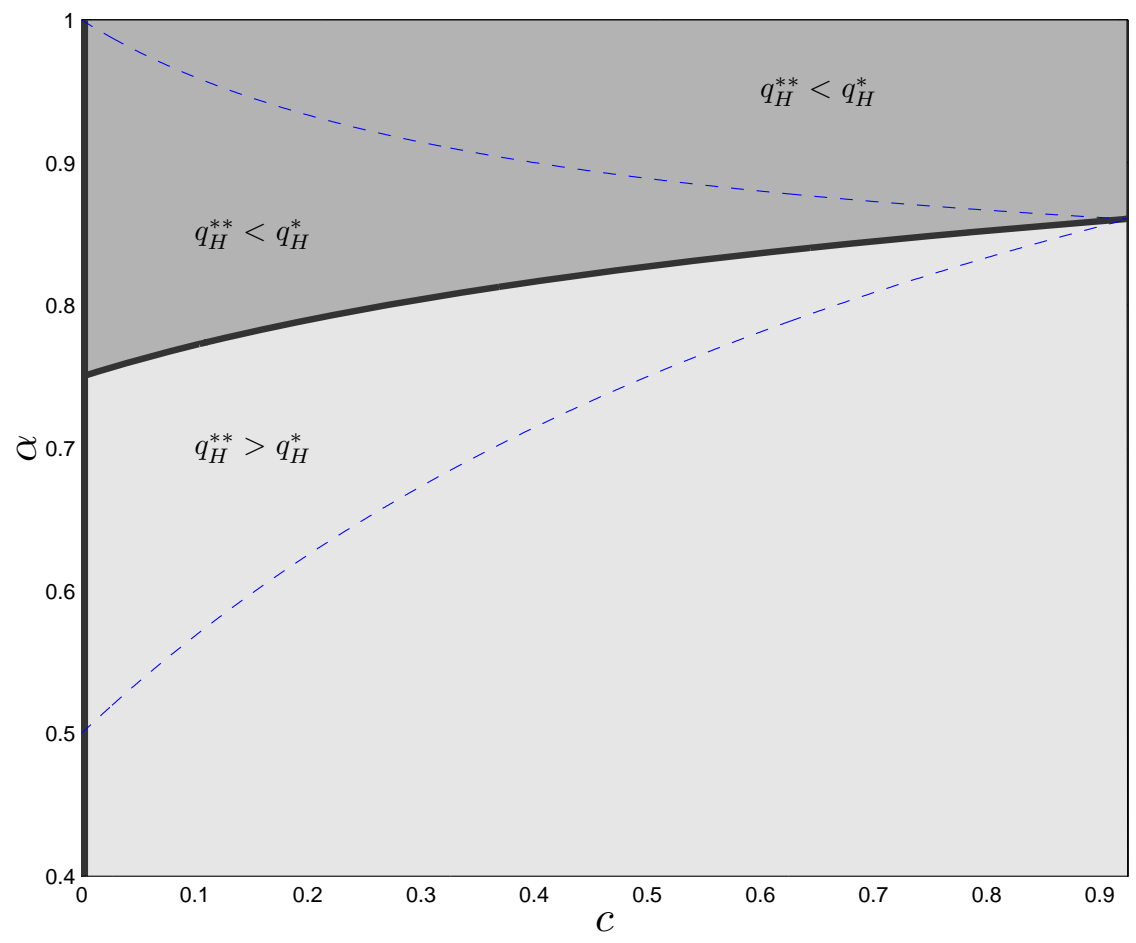

Figure 5: Efficiency for Buyer $H$ 's Usage Allowance. The area $q_{H}^{* *}<q_{H}^{*}$ regroups the set of pairs $\{\alpha, c\}$ for which the inefficiency decreases buyer $H$ 's usage allowance. The area $q_{H}^{* *}>q_{H}^{*}$ regroups the set of pairs $\{\alpha, c\}$ for which the inefficiency increases buyer $H$ 's usage allowance. The thick solid increasing concave line represents the case in which there is efficiency with congestion. There is also efficiency for the benchmark case of no congestion at $c=0$. 


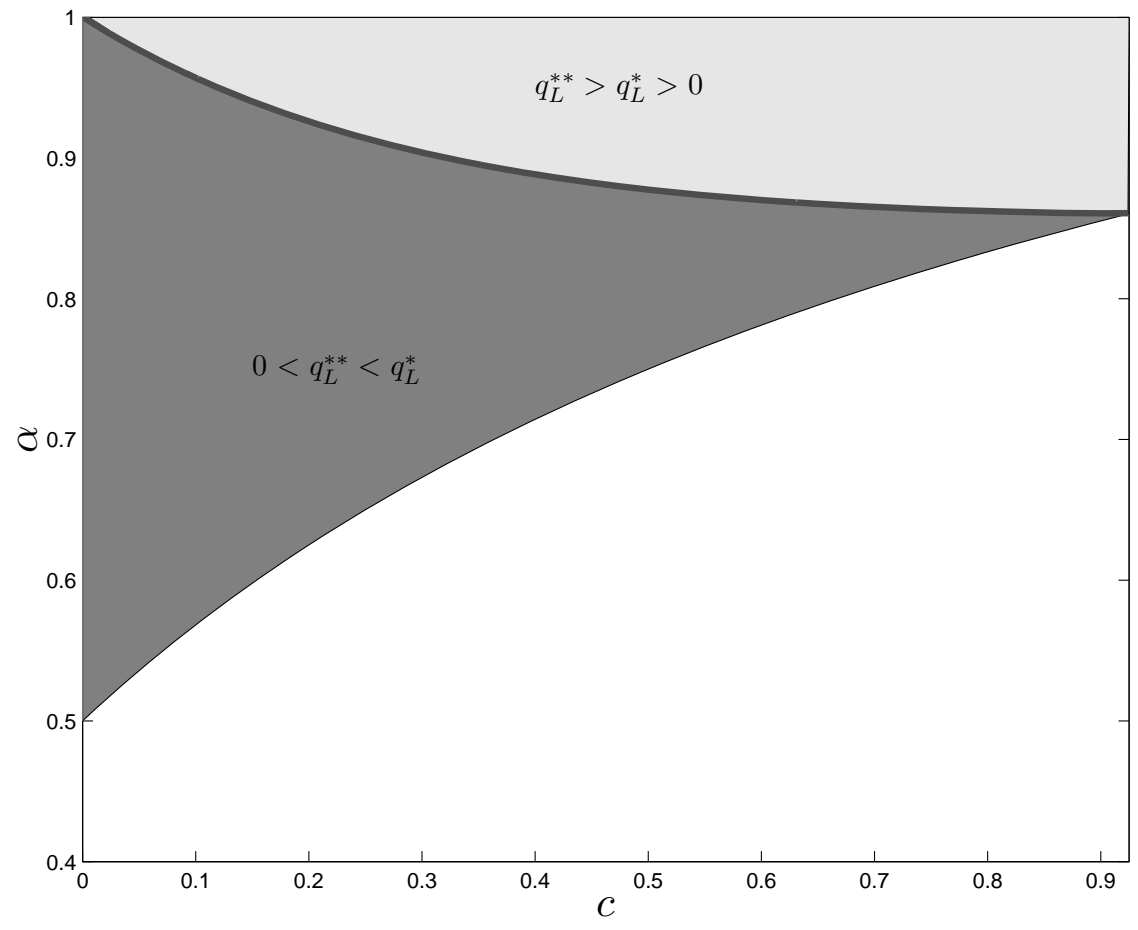

Figure 6: Efficiency for Buyer L's Usage Allowance. The area $q_{L}^{* *}>q_{L}^{*}>0$ regroups the set of pairs $\{\alpha, c\}$ for which the inefficiency increases buyer $L$ 's usage allowance. The area $0<q_{L}^{* *}<q_{L}^{*}$ regroups the set of pairs $\{\alpha, c\}$ for which the inefficiency decreases buyer $L$ 's usage allowance including the benchmark case of no congestion for $c=0$, and $\alpha \in(1 / 2,1)$. The thick solid decreasing convex line represents the case in which there is efficiency 
under second-degree price discrimination. Above the dotted increasing convex line, both buyers are offered positive usage allowances under perfect and second-degree price discrimination. Past the dotted decreasing concave line, as stated earlier, second-degree price discrimination implies that the seller offers the same package. The thick solid line represents the case in which the outcome is efficient.

\subsection{Consumer Surplus}

Having discussed the issue of efficiency by comparing first-degree and seconddegree price discrimination, we now examine whether price discrimination can enhance consumer surplus when there is congestion. To that end, we compare second-degree price discrimination with the case in which the seller does not price discriminate. See Appendix C for the full characterization and discussion of optimal packages under no price discrimination. We show that consumer surplus (through an increase in buyer $H$ 's consumer surplus since buyer $L$ 's consumer surplus is always zero regardless of price discrimination) may increase when the seller price discriminates. Figure 7 depicts the effect of price discrimination on consumer surplus for different values of $\alpha$ and c. The hat sign refers to the case of no price discrimination. Let $C S^{* *}=$ $u_{H}^{* *}-t_{H}^{* *}$ and $\widehat{C S}=\hat{u}_{H}-\hat{t}_{H}$ be the consumer surplus under second-degree price discrimination and no price discrimination, respectively. ${ }^{16}$

Remark 3.4 states that when the seller offers different packages to both buyers under second-degree price discrimination (recall Figure 4) and both buyers are serviced under no price discrimination (i.e., areas $\mathcal{A}_{2}$ and $\mathcal{A}_{3}$ in Figure 7 ), the effect on consumer surplus may be positive or negative. In areas $\mathcal{A}_{2}$ and $\mathcal{A}_{3}$, price discrimination yields an increase in buyer $H$ 's usage allowance and a decrease in buyer $L$ 's usage allowance, while the sum of the two usage allowances increases. The increase in usage allowance leads to an increase in buyer $H$ 's gross benefits (i.e., $u_{H}-t_{H}$ ). The increase in overall usage allowance leads to an increase as well in the congestion cost. In area $\mathcal{A}_{2}$, buyer $H$ 's gross benefit increases more than the congestion cost, and, thus,

\footnotetext{
${ }^{16}$ Recall that $u_{L}^{* *}-t_{L}^{* *}=\hat{u}_{L}-\hat{t}_{L}=0$.
} 


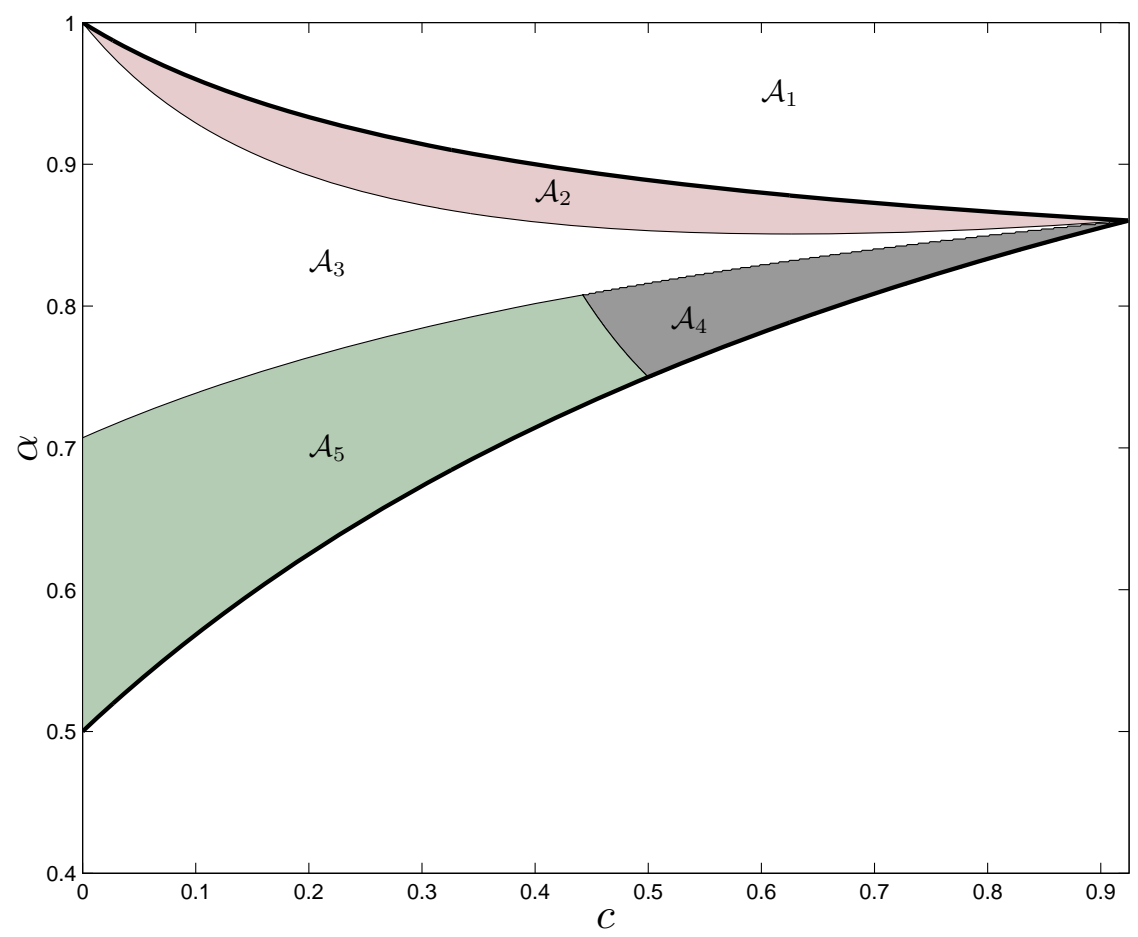

Figure 7: Effect of Price Discrimination on Consumer Surplus. For $i=$ $1,2,3,5$, area $\mathcal{A}_{i}$ regroups the set of pairs $\{\alpha, c\}$ for different cases for the effect of congestion on consumer surplus. Area $\mathcal{A}_{1}$ : Buyer $L$ is never excluded, $q_{H}^{* *}=$ $q_{L}^{* *}=\hat{q}$, and $C S^{* *}=\widehat{C S}$. Area $\mathcal{A}_{2}$ : Buyer $L$ is never excluded, $q_{H}^{* *}>\hat{q}>q_{L}^{* *}$, and $C S^{* *}>\widehat{C S}$. Area $\mathcal{A}_{3}$ : Buyer $L$ is never excluded, $q_{H}^{* *}>\hat{q}>q_{L}^{* *}$, and $C S^{* *}<\widehat{C S}$. Areas $\mathcal{A}_{4}$ and $\mathcal{A}_{5}$ : Buyer $L$ is excluded in the case of no price discrimination, $q_{H}^{* *}<\hat{q}, q_{L}^{* *}>0$, and $C S^{* *}>\widehat{C S}$. 
consumer surplus increases. In area $\mathcal{A}_{3}$, buyer $H$ 's gross benefit increases less than the congestion cost, and, thus, consumer surplus decreases.

Remark 3.4. From Figure 7, when both buyers are serviced under no price discrimination and the seller offers different packages under second-degree price discrimination, then price discrimination

1. increases consumer surplus in area $\mathcal{A}_{2}$, and

2. decreases consumer surplus in area $\mathcal{A}_{3}$.

Remark 3.5 states that the possibility to price discriminate may have no effect on consumer surplus. Since with congestion, the seller may decide to offer the same package to both buyers under second-degree price discrimination (Recall Figure 4), and, thus, there is no difference with the case of no price discrimination.

Remark 3.5. From Figure 7, when both buyers are serviced under no price discrimination, second-degree price discrimination has no effect on consumer surplus in area $\mathcal{A}_{1}$.

Remark 3.6 recalls the standard positive effect of price discrimination on consumer surplus when the seller excludes buyer $L$ under no price discrimination. This effect is present regardless of congestion.

Remark 3.6. From Figure 7, when buyer $L$ is excluded under no price discrimination, second-degree price discrimination increases consumer surplus in areas $\mathcal{A}_{4}$ and $\mathcal{A}_{5}$.

In area $\mathcal{A}_{4}$, the increase is due to an increase of the gross benefit and, if there is congestion, a decrease of the congestion cost. Here, buyer $L$ is offered a higher usage allowance while buyer $H$ 's usage allowance is reduced under second-degree price discrimination (compared to no price discrimination). Since the congestion cost borne by buyer $H$ is more affected by his own usage allowance than buyer $L$ 's usage allowance, congestion cost decreases for buyer $H$. The fee charged to buyer $H$ is also reduced so that gross benefit increases. In area $\mathcal{A}_{4}$, and in no price discrimination, buyer $L$ is 
excluded, and the seller extracts most of buyer $H$ 's surplus. Under seconddegree price discrimination, buyer $L$ is not excluded, and the seller captures less of buyer $H$ 's utility. The overall result in area $\mathcal{A}_{4}$ is an increase in the consumer surplus. In area $\mathcal{A}_{5}$, both gross benefit and congestion cost increase with price discrimination. In comparison with area $\mathcal{A}_{4}, \alpha$ is relatively low. Therefore, the variation of $q_{L}^{* *}$ and $q_{H}^{* *}$ observed in area $\mathcal{A}_{4}$ is attenuated in area $\mathcal{A}_{5} \cdot{ }^{17}$ As a consequence, the congestion cost increases. However, the increase in the gross benefit overcomes the increase in the congestion cost.

\section{Final Remarks}

In this paper, we provide an analysis of second-degree price discrimination for congestible network goods. We show that the seller does not always provide distinct contracts (i.e., it is not always optimal to price discriminate). We also show that congestion makes it impossible to obtain efficient allocations for any buyers. Finally, with congestion and for values of the parameters for which all types are serviced, consumer surplus under second-degree price discrimination may be greater than consumer surplus under no price discrimination. The existence of a region of the parameter space that generates consumer welfare-improving second-degree price discrimination, suggests room for additional empirical work studying demand for internet access and content. To date, with the exception of a few studies (Varian, 2001; Goolsbee and Klenow, 2006; Lambrecht et al., 2007), there is very little known about the efficiency properties of usage allowances and more generally 3-part tariff pricing schedules. Given the continual increasing importance of the internet, understanding and characterizing demand for internet content is an important first step to achieving efficiency in broadband networks.

As a first step in studying price discrimination of a congestible network good, we have abstracted from the buyer's usage decision once usage allowance is chosen and ignored a richer pricing scheme with a variable component linked to usage. ${ }^{18}$ Future research should consider a three-stage game

\footnotetext{
${ }^{17}$ Remember that $q_{L}^{* *}$ is increasing in $\alpha$ and $q_{H}^{* *}$ is decreasing in $\alpha$.

${ }^{18}$ As noted previously, usage allowance (e.g. allowance of 300GB per month) has been
} 
in which decisions on usage allowance and usage are split. In the first stage, the seller offers several internet packages. In the second stage, the buyers choose one of the two packages. In the third stage, the consumers interact strategically by choosing their usage levels. This would further our understanding on price discrimination when buyers choose both a type of service and a level of consumption.

shown empirically to increase usage (Varian, 2001). 


\section{A Second-Degree Price Discrimination}

In this appendix, we provide a proof of Proposition 2.3. Using (4) and (5), it follows that

$$
\begin{aligned}
& \alpha q_{L}-q_{L}^{2}-c q_{L}\left(q_{L}+q_{H}\right)-t_{L} \\
& +q_{H}-q_{H}^{2}-c q_{H}\left(q_{L}+q_{H}\right)-t_{H} \\
& \geq \alpha q_{H}-q_{H}^{2}-c q_{H}\left(q_{H}+q_{H}\right)-t_{H} \\
& +q_{L}-q_{L}^{2}-c q_{L}\left(q_{L}+q_{L}\right)-t_{L}
\end{aligned}
$$

so that

$$
\left(q_{H}-q_{L}\right)(1-\alpha)+c\left(q_{H}-q_{L}\right)^{2} \geq 0 .
$$

If $c=0$, then, from (16), $q_{H} \geq q_{L}$. If $c>0$, then we need to consider three cases. 1) If $q_{H}>q_{L}$, then (16) implies that

$$
q_{H}>q_{L}-\frac{1-\alpha}{c}
$$

which is always true when $q_{H} \geq q_{L}$. 2) If $q_{H}=q_{L}$, then (16) holds. 3) If $q_{H}<q_{L}$, then (16) implies that the condition

$$
q_{H}+\frac{1-\alpha}{c}<q_{L}
$$

must hold as well.

1. Suppose first that $q_{H}>q_{L}$.

(a) At the optimum, (6) is active. To see this, using (5), $\alpha \in(0,1)$, and $q_{H}>q_{L}$, it follows that

$$
\begin{aligned}
q_{H}-q_{H}^{2}-c q_{H}\left(q_{L}+q_{H}\right)-t_{H} & \geq q_{L}-q_{L}^{2}-c q_{L}\left(q_{L}+q_{L}\right)-t_{L} \\
& \geq \alpha q_{L}-q_{L}^{2}-c q_{L}\left(q_{L}+q_{H}\right)-t_{L}
\end{aligned}
$$


Suppose to the contrary that (6) is inactive, i.e., $\alpha q_{L}-q_{L}^{2}-c q_{L}\left(q_{L}+\right.$ $\left.q_{H}\right)-t_{L}>0$, then so is $(7)$. This cannot be an optimum since $t_{L}$ and $t_{H}$ can be increased without any effect on incentive compatibility and individual rationality. Hence, (6) is active, i.e.,

$$
\begin{aligned}
t_{L} & =\alpha q_{L}-q_{L}^{2}-c q_{L}\left(q_{L}+q_{H}\right), \\
& =\alpha q_{L}-(1+c) q_{L}^{2}-c q_{L} q_{H}
\end{aligned}
$$

at the optimum.

(b) At the optimum, (5) is active. To see this, using (19) and (22),

$$
\begin{aligned}
q_{H}-q_{H}^{2}-c q_{H}\left(q_{L}+q_{H}\right)-t_{H} & \geq q_{L}-q_{L}^{2}-c q_{L}\left(q_{L}+q_{L}\right)-t_{L} \\
& \geq \alpha q_{L}-q_{L}^{2}-c q_{L}\left(q_{L}+q_{H}\right)-t_{L}=0 .
\end{aligned}
$$

Suppose to the contrary that (5) is inactive, i.e., $q_{H}-q_{H}^{2}-c q_{H}\left(q_{L}+\right.$ $\left.q_{H}\right)-t_{H}>q_{L}-q_{L}^{2}-c q_{L}\left(q_{L}+q_{L}\right)-t_{L}$, then $t_{H}$ can be increased without any effect on incentive compatibility or individual rationality. Hence, from (5),

$$
q_{H}-q_{H}^{2}-c q_{H}\left(q_{L}+q_{H}\right)-t_{H}=q_{L}-q_{L}^{2}-c q_{L}\left(q_{L}+q_{L}\right)-t_{L},
$$

so that

$$
t_{H}=q_{H}-(1+c) q_{H}^{2}-(1-\alpha) q_{L}+c q_{L}^{2}-2 c q_{H} q_{L}
$$

at the optimum.

(c) Expressions (4) and (7) can be neglected. 
(d) Plugging (22) and (26) into (3) yields

$$
\begin{aligned}
\Pi^{* *}(\alpha, c)= & \max _{q_{L}, q_{H} \geq 0} \alpha q_{L}-(1+c) q_{L}^{2}-c q_{L} q_{H}+q_{H}-(1+c) q_{H}^{2} \\
& -(1-\alpha) q_{L}+c q_{L}^{2}-2 c q_{H} q_{L}, \\
= & \max _{q_{L}, q_{H} \geq 0}(2 \alpha-1) q_{L}-q_{L}^{2}+q_{H}-(1+c) q_{H}^{2}-3 c q_{H} q_{L} .
\end{aligned}
$$

We consider two subcases.

- Suppose first that $\alpha \in\left(\frac{2+5 c}{4(1+c)}, \frac{2(1+2 c)}{2+5 c}\right)$. Then, the first-order conditions are ${ }^{19}$

$$
\begin{gathered}
\frac{\partial}{\partial q_{L}}: 2 \alpha-1-2 q_{L}-3 c q_{H}=0 \\
\frac{\partial}{\partial q_{H}}: 1-2(1+c) q_{H}-3 c q_{L}=0 .
\end{gathered}
$$

Solving (30) and (31) yields (9) and (10) such that $q_{H}^{* *}>q_{L}^{* *}>$ 0 when $\alpha \in\left(\frac{2+5 c}{4(1+c)}, \frac{2(1+2 c)}{2+5 c}\right){ }^{20}$

- Suppose next that $\alpha \in\left(0, \frac{2+5 c}{4(1+c)}\right]$. Then, $q_{L}^{* *}=0$, and, from (28) evaluated at $q_{L}=0$, the first-order condition is

$$
\frac{\partial}{\partial q_{H}}: 1-2(1+c) q_{H}=0
$$

which yields $q_{H}^{* *}=\frac{1}{2(1+c)}$, so that, using $(26), t_{H}^{* *}=\frac{1}{4(1+c)}$, as in $(8)$

2. Before considering the case $\alpha \in\left[\frac{2(1+2 c)}{2+5 c}, 1\right)$, we show that $q_{H}<q_{L}$ is not possible in equilibrium. Suppose to the contrary that $q_{H}<q_{L}$.

${ }^{19}$ Given Assumption 2.2, the Hessian matrix

$$
\mathcal{H}=\left[\begin{array}{cc}
-2 & -3 c \\
-3 c & -2(1+c)
\end{array}\right]
$$

is negative definite.

${ }^{20}$ Here, $\alpha>\frac{2+5 c}{4(1+c)}$ implies that $q_{L}^{* *}>0$, while $\alpha<\frac{2(1+2 c)}{2+5 c}$ implies that $q_{H}^{* *}>q_{L}^{* *}$. 
(a) At the optimum, (7) is active. To see this, using (4) and the fact that $\alpha \in(0,1)$ and that $(18)$ must hold in this case, it follows that

$$
\begin{aligned}
\alpha q_{L}-q_{L}^{2}-c q_{L}\left(q_{L}+q_{H}\right)-t_{L} & \geq \alpha q_{H}-q_{H}^{2}-c q_{H}\left(q_{H}+q_{H}\right)-t_{H}, \\
& >q_{H}-q_{H}^{2}-c q_{H}\left(q_{H}+q_{L}\right)-t_{H} .
\end{aligned}
$$

Suppose to the contrary that (7) is inactive, i.e., $q_{H}-q_{H}^{2}-c q_{H}\left(q_{H}+\right.$ $\left.q_{L}\right)-t_{H}>0$, then so is (6). This cannot be an optimum since $t_{L}$ and $t_{H}$ can be increased without any effect on incentive compatibility and individual rationality. Hence, (7) is active, i.e.,

$$
\begin{aligned}
t_{H} & =q_{H}-q_{H}^{2}-c q_{H}\left(q_{H}+q_{L}\right), \\
& =q_{H}-(1+c) q_{H}^{2}-c q_{L} q_{H}
\end{aligned}
$$

at the optimum.

(b) At the optimum, (4) is active. To see this, using (34) and (36),

$$
\begin{aligned}
\alpha q_{L}-q_{L}^{2}-c q_{L}\left(q_{L}+q_{H}\right)-t_{L} & \geq \alpha q_{H}-q_{H}^{2}-c q_{H}\left(q_{H}+q_{H}\right)-t_{H}, \\
& \geq q_{H}-q_{H}^{2}-c q_{H}\left(q_{H}+q_{L}\right)-t_{H}=0 .
\end{aligned}
$$

Suppose to the contrary that (4) is inactive, i.e., $\alpha q_{L}-q_{L}^{2}-c q_{L}\left(q_{L}+\right.$ $\left.q_{H}\right)-t_{L}>\alpha q_{H}-q_{H}^{2}-c q_{H}\left(q_{H}+q_{H}\right)-t_{H}$, then $t_{L}$ can be increased without any effect on incentive compatibility or individual rationality. Hence,

$$
\alpha q_{L}-q_{L}^{2}-c q_{L}\left(q_{L}+q_{H}\right)-t_{L}=\alpha q_{H}-q_{H}^{2}-c q_{H}\left(q_{H}+q_{H}\right)-t_{H},
$$

so that

$$
t_{L}=\alpha q_{L}-(1+c) q_{L}^{2}+(1-\alpha) q_{H}+c q_{H}^{2}-2 c q_{L} q_{H} .
$$


(c) Plugging (36) and (40) into (3) yields

$$
\begin{aligned}
\Pi^{* *}(\alpha, c)= & \max _{q_{L}, q_{H}} \alpha q_{L}-(1+c) q_{L}^{2}+(1-\alpha) q_{H}+c q_{H}^{2}-2 c q_{L} q_{H} \\
& +q_{H}-(1+c) q_{H}^{2}-c q_{L} q_{H}, \\
= & \max _{q_{L}, q_{H}} \alpha q_{L}-(1+c) q_{L}^{2}+(2-\alpha) q_{H}-q_{H}^{2}-3 c q_{L} q_{H} .
\end{aligned}
$$

We consider two subcases.

- Suppose first that $q_{L}^{* *}>q_{H}^{* *}>0$. Then, the first-order conditions are

$$
\begin{aligned}
& \frac{\partial}{\partial q_{L}}: \alpha-2(1+c) q_{L}-3 c q_{H}=0 \\
& \frac{\partial}{\partial q_{H}}: 2-\alpha-2 q_{H}-3 c q_{L}=0
\end{aligned}
$$

so that

$$
\begin{aligned}
q_{L}^{* *} & =\frac{2 \alpha-3(2-\alpha) c}{4(1+c)-9 c^{2}} \\
q_{H}^{* *} & =\frac{2(2-\alpha)+(4-5 \alpha) c}{4(1+c)-9 c^{2}} .
\end{aligned}
$$

Given (18), we need

$$
q_{H}^{* *}+\frac{1-\alpha}{c}<q_{L}^{* *}
$$

or $8(1-\alpha) c+(1+\alpha) c^{2}+4(1-\alpha)<0$, which is impossible.

- Suppose next that $q_{L}^{* *}>q_{H}^{* *}=0$. Then, the first-order condition is

$$
\frac{\partial}{\partial q_{L}}: \alpha-2(1+c) q_{L}=0
$$

so that

$$
q_{L}^{* *}=\frac{\alpha}{2(1+c)}
$$


and thus profit is

$$
\Pi^{* *}(\alpha, c)=\frac{\alpha^{2}}{4(1+c)} .
$$

However, this cannot be an optimum since the solution $q_{H}^{* *}=$ $\frac{1}{2(1+c)}, q_{L}^{* *}=0, t_{L}^{* *}=0$ and $t_{H}^{* *}=\frac{1}{4(1+c)}$ yields profit $\frac{1}{4(1+c)}$ that is strictly greater than (50).

3. Suppose finally that $\alpha \in\left[\frac{2(1+2 c)}{2+5 c}, 1\right)$. Since $q_{H}^{* *}>q_{L}^{* *}$ cannot hold, and $q_{H}^{* *}<q_{L}^{* *}$ is not possible, it must be that $q_{H}^{* *}=q_{L}^{* *}$. Hence, (3) is rewritten as

$$
\Pi^{* *}(\alpha, c)=\max _{q} 2\left(\alpha q-(1+2 c) q^{2}\right),
$$

which yields (11). 


\section{B First-Degree Price Discrimination}

In this appendix, we state and prove the optimal contract under first-degree (perfect) price discrimination. Suppose that the seller can perfectly price discriminate. Hence, he solves the following program

$$
\Pi^{*}(\alpha, c)=\max _{q_{L}, q_{H}, t_{L}, t_{H} \geq 0} t_{L}+t_{H},
$$

subject to the IR (individual rationality) constraints

$$
\begin{aligned}
& (\underline{I R}): \alpha q_{L}-q_{L}^{2}-c\left(q_{L}+q_{H}\right) q_{L} \geq t_{L}, \\
& (\overline{I R}): q_{H}-q_{H}^{2}-c\left(q_{L}+q_{H}\right) q_{H} \geq t_{H} .
\end{aligned}
$$

Proposition B.1 provides the optimal packages under perfect price discrimination.

Proposition B.1. Under perfect price discrimination, $q_{H}^{*}>q_{L}^{*} \geq 0$ and $t_{H}^{*}>t_{L}^{*} \geq 0$, such that

1. For $\alpha \in\left(0, \frac{c}{1+c}\right],\left\{q_{L}^{*}, t_{L}^{*}\right\}=\{0,0\}$ and

$$
\left\{q_{H}^{*}, t_{H}^{*}\right\}=\left\{\frac{1}{2(1+c)}, \frac{1}{4(1+c)}\right\}
$$

and

2. For $\alpha \in\left(\frac{c}{1+c}, 1\right)$,

$$
\begin{aligned}
\left\{q_{L}^{*}, t_{L}^{*}\right\} & =\left\{\frac{\alpha-(1-\alpha) c}{2(1+2 c)}, \frac{\alpha^{2}-(1-\alpha) \alpha c}{4(1+2 c)}\right\}, \\
\left\{q_{H}^{*}, t_{H}^{*}\right\} & =\left\{\frac{1+(1-\alpha) c}{2(1+2 c)}, \frac{1+(1-\alpha) c}{4(1+2 c)}\right\} .
\end{aligned}
$$

Proof. Since both (53) and (54) are active, (52) is rewritten as

$$
\Pi^{*}(\alpha, c)=\max _{q_{L}, q_{H} \geq 0} \alpha q_{L}-q_{L}^{2}+q_{H}-q_{H}^{2}-c\left(q_{L}+q_{H}\right)^{2} .
$$


1. Suppose first that $\alpha \in\left(0, \frac{c}{1+c}\right]$. Then, $q_{L}^{*}=0$, and, thus $t_{L}^{*}=0$. From (58) evaluated at $q_{L}=0$, the first-order condition is

$$
\frac{\partial}{\partial q_{H}}: 1-2 q_{H}-2 c q_{H}=0
$$

which yields $q_{H}=\frac{1+(1-\alpha) c}{2(1+2 c)}$ and $t_{H}^{*}=\frac{1+(1-\alpha) c}{4(1+2 c)}$, as in (55).

2. Suppose next that $\alpha \in\left(\frac{c}{1+c}, 1\right)$. Then, the unique interior solution is characterized by the first-order conditions ${ }^{21}$

$$
\begin{aligned}
& \frac{\partial}{\partial q_{L}}: \alpha-2 q_{L}-2 c\left(q_{L}+q_{H}\right)=0, \\
& \frac{\partial}{\partial q_{H}}: 1-2 q_{H}-2 c\left(q_{L}+q_{H}\right)=0 .
\end{aligned}
$$

Solving (60) and (61) yields $q_{L}^{*}$ and $q_{H}^{*}$ as in (56) and (57) such that $q_{L}^{*}>0$ when $\alpha \in\left(\frac{c}{1+c}, 1\right)$. Plugging back $q_{L}^{*}$ and $q_{H}^{*}$ into (53) and (54) yields $t_{L}^{*}$ and $t_{H}^{*}$ as in (56) and (57).

${ }^{21}$ The Hessian matrix $\mathcal{H}=-2\left[\begin{array}{cc}1+c & c \\ c & 1+c\end{array}\right]$ is negative definite. 


\section{No Price Discrimination}

Suppose that the seller cannot price discriminate among the two buyers. Then, he proposes the same package $\{q, t\}$ to both buyers, and he solves the following program:

$$
\hat{\Pi}=\max _{q, t \geq 0} t 1_{\left[u_{L}(q, q) \geq t\right]}+t 1_{\left[u_{H}(q, q) \geq t\right]}
$$

Here, $1_{[\cdot]}$ is an indicator function equal to one if the statement in [.] is true, and zero otherwise.

Proposition C.1 provides the optimal solutions for the case in which the seller cannot price discriminate. For low values of $\alpha$, the low-valuation buyer is excluded. Because the low-valuation buyer does not value the good ( $\alpha$ is low), he will not be disposed to pay a high flat fee. As a consequence, selling to the low-valuation buyer will not be profitable for the seller. For high values of $\alpha$, both buyers accept to consume the good. The presence of a congestion cost renders first-degree price discrimination more difficult. Specifically, as heterogeneity between the two buyers increases (i.e., a decrease in $\alpha$ ), the seller finds it more costly to offer a non-zero contract to the low-valuation buyer. The reason is that offering an extra unit of usage to the low-valuation buyer yields extra revenue that is dominated by the extra congestion cost. An increase in the cost of congestion makes it more likely for the low-valuation buyer to be excluded as well.

Proposition C.1. Suppose the seller cannot price discriminate.

1. For $\alpha \in\left(0, \sqrt{\frac{1+2 c}{2(1+c)}}\right]$, the seller excludes the low-valuation buyer and service the high-valuation buyer by offering quality

$$
\hat{q}=\frac{1}{2(1+c)}
$$

for a fee

$$
\hat{t}=\frac{1}{4(1+c)}
$$


The seller's payoff (or social welfare) is

$$
\hat{\Pi}=\hat{W}=\frac{1}{4(1+c)} .
$$

2. For $\alpha \in\left(\sqrt{\frac{1+2 c}{2(1+c)}}, 1\right)$, the seller services both buyers by offering quality

$$
\hat{q}=\frac{\alpha}{2(1+2 c)}
$$

for a fee

$$
\hat{t}=\frac{\alpha^{2}}{4(1+2 c)}
$$

The seller's payoff is

$$
\hat{\Pi}=\frac{\alpha^{2}}{2(1+2 c)}
$$

while welfare is

$$
\hat{W}=\frac{\alpha}{2(1+2 c)}
$$

Proof. We solve the problem piecewise. The seller will not propose $\{q, t\}$ such that $u_{L}(q, q)<t$ and $u_{H}(q, q)<t$. We then distinguish two cases: $u_{L}(q, q)<t \leq u_{H}(q, q)$ and $t \leq u_{L}(q, q)<u_{H}(q, q)$.

1. Case 1: $u_{L}(q, q)<t \leq u_{H}(q, q)$. The low-valuation buyer does not accept the offer, so that the congestion cost is simply $c q^{2}$, then the utility of the high-valuation buyer is $u_{H}(q, q)=q-q^{2}-c q^{2} \geq t$. At the optimum, $u_{H}(q, q)=t$ because if $u_{H}(q, q)>t$ the seller can increase $t$ without affecting the constraints. The seller's program is rewritten as

$$
\hat{\Pi}=\max _{q \geq 0} q-q^{2}-c q^{2}
$$

so that the first-order condition is $\frac{\partial}{\partial q}: 1-2 q-2 c q=0$, which yields

$$
\hat{q}=\frac{1}{2(1+c)}
$$

The buyers' utilities are $u_{L}(\hat{q}, \hat{q})=0$ and $u_{H}(\hat{q}, \hat{q})=\hat{t}=\frac{1}{4(1+c)}$. The 
seller's profit $\hat{\Pi}$ and the social welfare $\hat{W}$ are

$$
\hat{\Pi}=\hat{W}=\frac{1}{4(1+c)}
$$

2. Case 2: $t \leq u_{L}(q, q)<u_{H}(q, q)$. Both buyers accept the package $\{q, t\}$. $u_{L}(q, q)=\alpha q-q^{2}-c(q+q) q$, and $u_{H}(q, q)=q-q^{2}-c(q+q) q$. At the optimum, $u_{L}(q, q)=t$ because if $u_{L}(q, q)>t$ the seller can increase $t$ without affecting the constraints. The seller's program is rewritten as

$$
\hat{\Pi}=\max _{q \geq 0} 2\left(\alpha q-q^{2}-c q(q+q)\right)
$$

so that the first-order condition is $\frac{\partial}{\partial q}: \alpha-2(1+2 c) q=0$, which yields

$$
\hat{q}=\frac{\alpha}{2(1+2 c)}
$$

Hence,

$$
\hat{\Pi}=\frac{\alpha^{2}}{2(1+2 c)} .
$$

and

$$
\begin{aligned}
\hat{W} & =\hat{u}_{H}+\hat{u}_{L}, \\
& =\frac{\alpha}{2(1+2 c)} .
\end{aligned}
$$

3. It follows that the low-valuation buyer is excluded if and only if (72) is greater than $(75)$, i.e., $\alpha \in\left(0, \sqrt{\frac{1+2 c}{2(1+c)}}\right]$. 


\section{Figures}

Figures 8 and 9 provide information about the partial effects of $\alpha$ and $c$, respectively, on optimal usage allowance under second-degree price discrimination, holding constant the other parameter.

Figures 10 and 11 provide a general three-dimensional view of the difference in usage allowance under perfect price discrimination and second-degree price discrimination. 

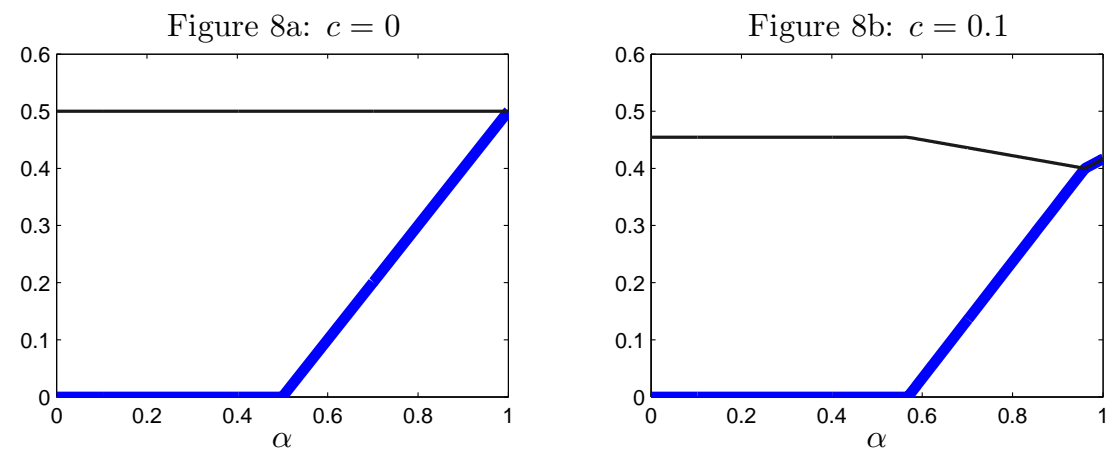

Figure 8c: $c=0.5$

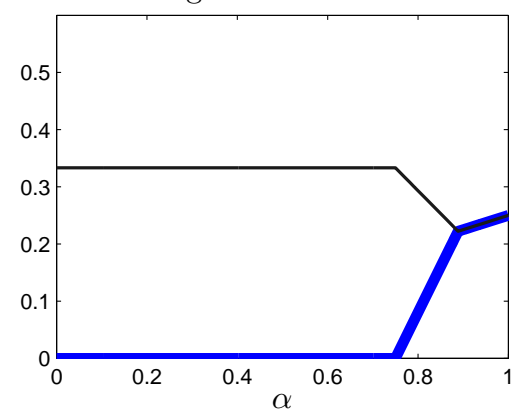

Figure 8d: $c=0.9$

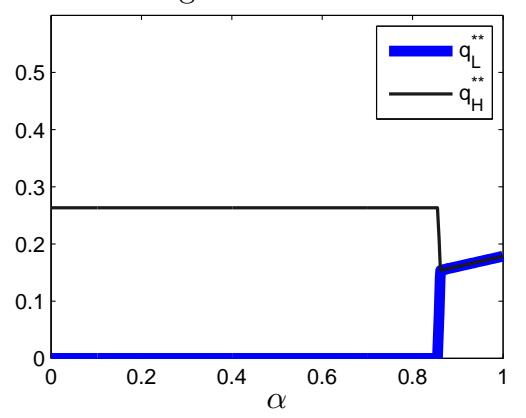

Figure 8: Effect of Buyer L's Valuation on Usage Allowances. The optimal usage allowances $q_{L}^{* *}$ and $q_{H}^{* *}$ under second-degree price discrimination are plotted as functions of $\alpha \in[0,1]$ for different values of $c \in\{0,0.1,0.5,0.9\}$. An increase in $\alpha$ reduces the difference in usage allowances between the two types of buyers. 
Figure 9a: $\alpha=0.5$

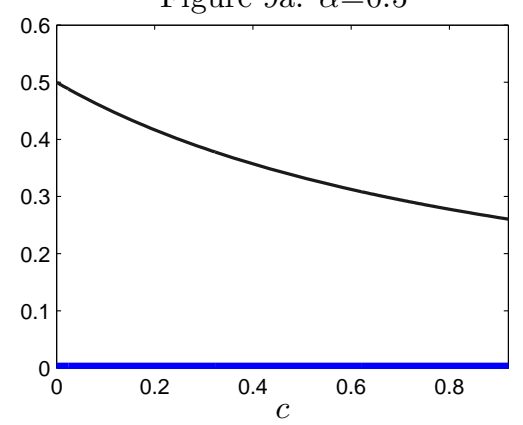

Figure 9c: $\alpha=0.7$

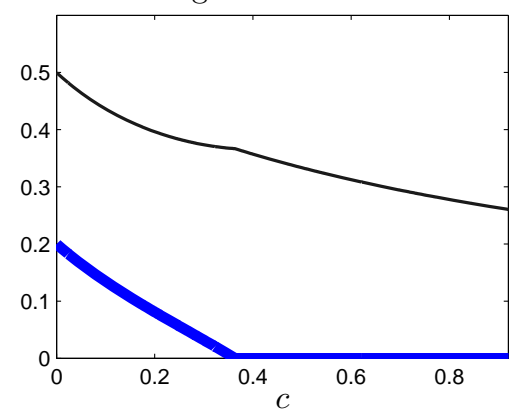

Figure 9b: $\alpha=0.6$

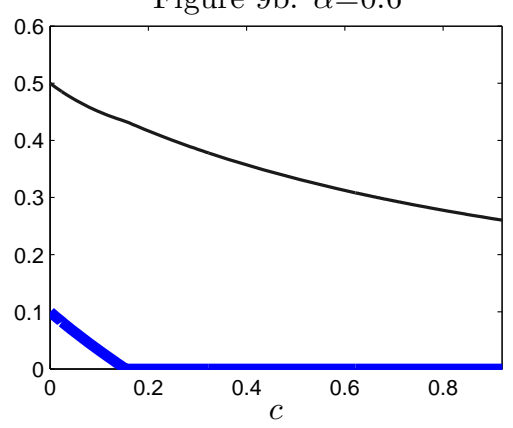

Figure 9d: $\alpha=0.9$

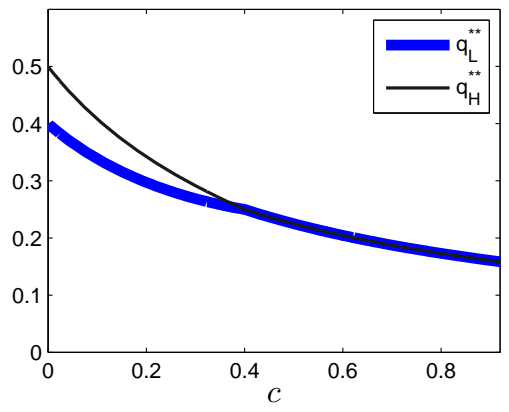

Figure 9: Effect of Congestion Cost on Usage Allowances. The optimal usage allowances $q_{L}^{* *}$ and $q_{H}^{* *}$ under second-degree price discrimination are plotted as functions of $c \in[0, \bar{c}]$ for different values of $\alpha \in\{0.5,0.6,0.7,0.9\}$. An increase in $c$ reduces the difference in usage allowances between the two types of buyers. 


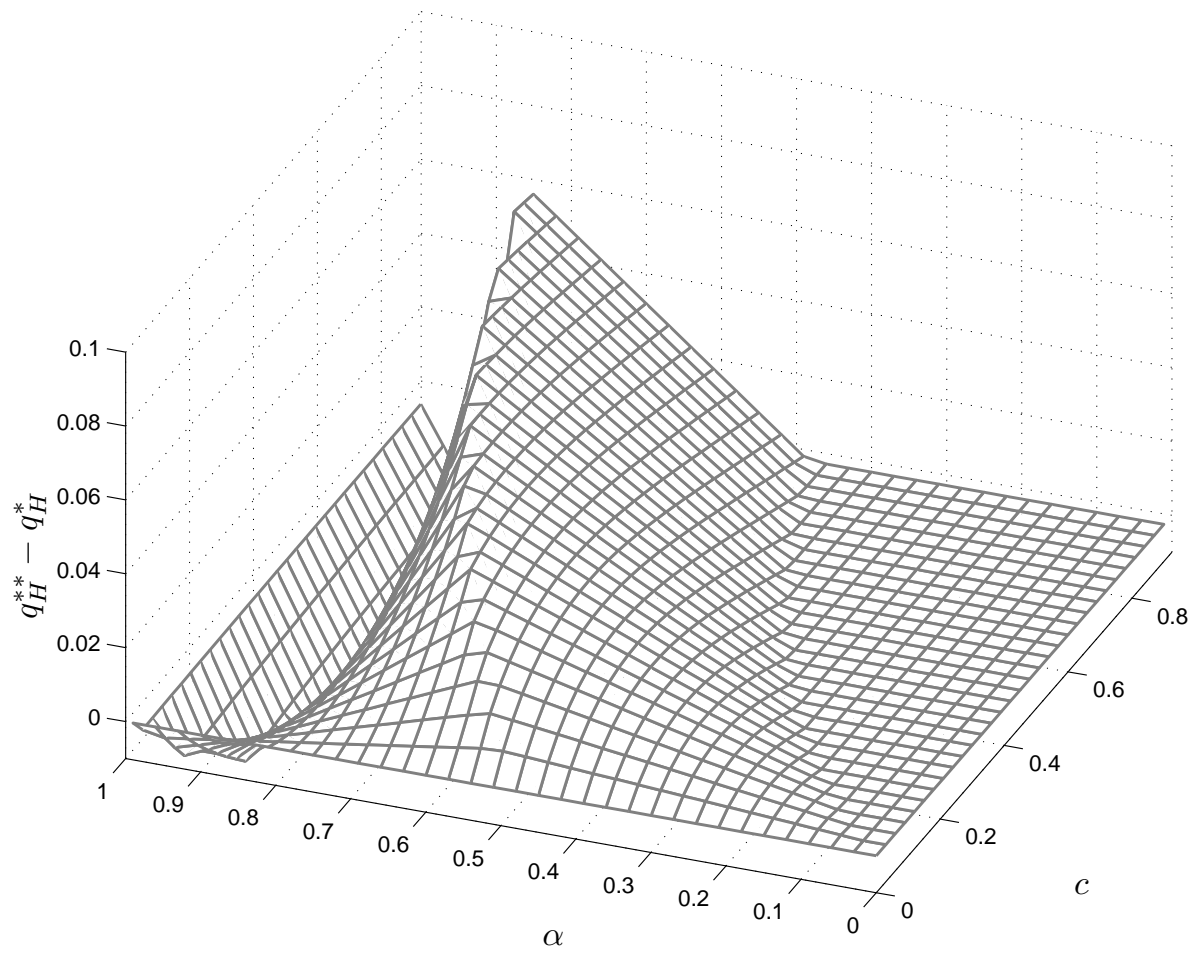

Figure 10: Efficiency for Buyer $H$ 's Usage Allowance, 3D. A three-dimensional view of the difference in buyer $H$ 's usage allowances between second-degree and perfect price discrimination is provided. That is, $q_{H}^{* *}-q_{H}^{*}$ is plotted for all values of $\alpha$ and $c$. 


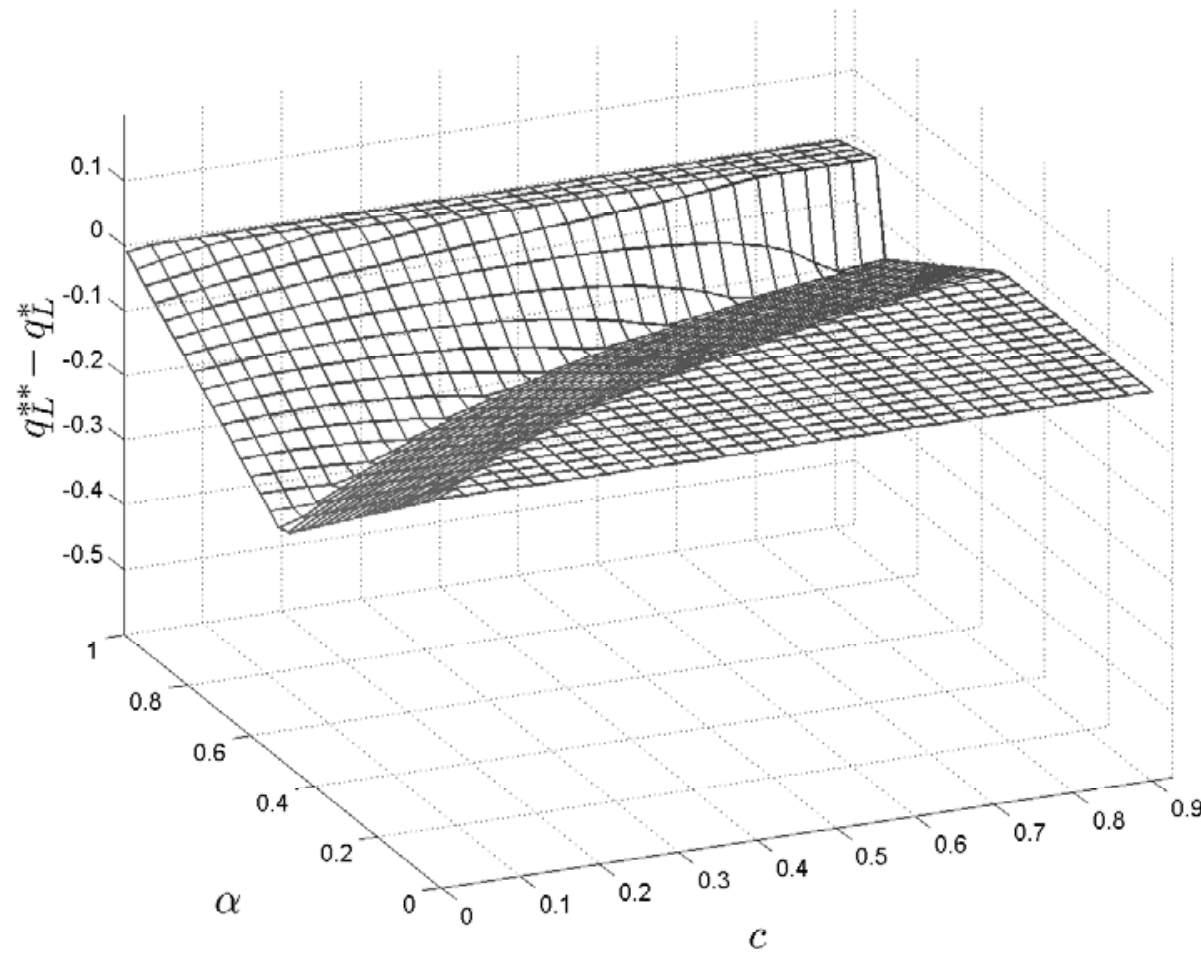

Figure 11: Efficiency for Buyer L's Usage Allowance, 3D. A three-dimensional view of the difference in buyer $L$ 's usage allowances between second-degree and perfect price discrimination is provided. That is, $q_{L}^{* *}-q_{L}^{*}$ is plotted for all values of $\alpha$ and $c$. 


\section{References}

R.N. Clarke. Costs of Neutral/Unmanaged IP Networks. Rev. Network Econ., 8(1):61-89, 2009.

A.I. Czerny and A. Zhang. Airport Congestion Pricing when Airlines Price Discriminate. Working paper 12-02, WHU-Otto Beisheim School of Management, 2012.

A. Goolsbee and P.J. Klenow. Valuing Consumer Products by the Time Spent Using Them: An Application to the Internet. Amer. Econ. Rev., 96 (2):108-113, 2006.

T.J. Holmes. The Effects of Third-Degree Price Discrimination in Oligopoly. Amer. Econ. Rev., 79(1):244-250, 1989.

A. Lambrecht, K. Seim, and B. Skiera. Does Uncertainty Matter? Consumer Behavior under Three-Part Tariffs. Marketing Sci., 26(5):698-710, 2007.

J. Robinson. The Economics of Imperfect Competition. Macmillan, 1933.

R Schmalensee. Output and Welfare Implications of Monopolistic ThirdDegree Price Discrimination. Amer. Econ. Rev., 71(1):242-247, 1981.

H.R. Varian. Price Discrimination and Social Welfare. Amer. Econ. Rev., 75 (4):970-875, 1985.

H.R. Varian. The Demand for Bandwidth: Evidence from the INDEX Project. In R.W. Crandall and J.H. Alleman, editors, Broadband: Should We Regulate High-Speed Internet Access?, pages 39-56. Brookings Institution Press, 2001. 